to RTX $(6,6)$. The demographic and response data DAS 28 are shown in Table 1. There are no differences in the years of evolution, $\%$ of women, FR or ACPA positive, erosions and disease activity, as measured by DAS 28 , before the switch, between The two groups (anti-TNF vs non-anti-TNF). Patients in the anti-TNF group were slightly younger than non-anti-TNF.When the DAS 28 response is evaluated at 3 and 6 months, modifying the treatment is effective (DAS 28 beginning 4,40 vs DAS 286 months $2.8 p<0.001$ ). When assessing the response to change, there is no difference in the DAS 28 response at 3 months or 6 months, if you switched to anti-TNF or non-anti-TNF ( 3.18 vs $2.52 \mathrm{p}=0.122$ ).

When comparing the patients with anti TNF alpha vs TCZ, $62.5 \%$ of the patients with TCZ are in remission compared to $38,5 \%(p=0.047)$.

Table 1

\begin{tabular}{lcccc}
\hline & $\begin{array}{c}\text { Total } \\
\mathrm{n}=61\end{array}$ & $\begin{array}{c}\text { Swich anti-TNF alpha } \\
\mathrm{n}=26\end{array}$ & $\begin{array}{c}\text { Swich No anti-TNF alpha } \\
\mathrm{n}=35\end{array}$ & $\mathrm{p}$ \\
\hline Age (years) & $60.08 \pm 11,5$ & $56.19 \pm 8,4$ & $62.97 \pm 12,8$ & 0.023 \\
Sex (\% M) & 80.3 & 84.6 & 77.1 & 0.532 \\
Years evolution (years) & $17.44 \pm 9,4$ & $18.92 \pm 11,6$ & $16.34 \pm 7,4$ & 0.298 \\
Erosion (\%) & 90.2 & 92.3 & 88.6 & 1.000 \\
FR+ (\%) & 77.0 & 76.9 & 77.1 & 1.000 \\
ACPA+ $(\%)$ & 91.8 & 88.5 & 94.3 & 0.642 \\
\hline
\end{tabular}

Table 2

\begin{tabular}{lcccc}
\hline & $\begin{array}{c}\text { Total } \\
\mathrm{n}=61\end{array}$ & $\begin{array}{c}\text { Swich anti-TNF alpha } \\
\mathrm{n}=26\end{array}$ & $\begin{array}{c}\text { Swich No anti-TNF alpha } \\
\mathrm{n}=35\end{array}$ & $\mathrm{p}$ \\
\hline DAS 28 Swich & $4.40 \pm 1,38$ & $4.61 \pm 1,5$ & $4.21 \pm 1,3$ & 0.216 \\
DAS 28 3 months & $3.18 \pm 1,52$ & $3.52 \pm 1,09$ & $2.96 \pm 1,7$ & 0.167 \\
DAS 28 6 months & $2.8 \pm 1,54$ & $3.18 \pm 1,74$ & $2.52 \pm 1,3$ & 0.122 \\
DAS 28 <2.6 6 m (\%) & 52.5 & 38.5 & 62.5 & 0.047 \\
\hline
\end{tabular}

Conclusions: In this retrospective study in daily clinical practice, it is evident that the change in treatment after failure of the first biological one, without differences if the change is to an anti TNF or another treatment. The percentage of patients who are remission at 6 months is higher if the change is at TCZ. Given the small number of patients, larger studies would be needed to confirm the results. References:

[1] Johnston SS et col. Comparison of Biologic Disease-Modifying Antirheumatic Drug Therapy Persistence Between Biologics Among Rheumatoid Arthritis Patients Switching from Another Biologic. Rheumatol Ther.

Disclosure of Interest: None declared

DOI: 10.1136/annrheumdis-2017-eular.3375

\section{AB0382 OUTCOMES OF ETANERCEPT THERAPY IN ELDERLY RHEUMATOID ARTHRITIS PATIENTS: AN INVESTIGATION OF THE AKITA ORTHOPEDIC GROUP ON RHEUMATOID ARTHRITIS REGISTRY}

${ }_{\text {H. Aonuma }}{ }^{1}$, T. Kashiwagura ${ }^{2}$, M. Urayama ${ }^{3}$, M. Kobayashi $^{4}$, T. Aizawa $^{5}$ Y. Sugimura ${ }^{6}$, N. Miyakoshi ${ }^{7}$, Y. Shimada ${ }^{7} .{ }^{1}$ Orthopedic Surgery, Kakunodate Municipal Hospital, Senboku; ${ }^{2}$ Orthopedic Surgery, Akita City Hospital, Akita; ${ }^{3}$ Orthopedic Surgery, Ogachi Central Hospital, Yuzawa; ${ }^{4}$ Orthopedic Surgery, Hiraka General Hospital, Yokote; ${ }^{5}$ Orthopedic Surgery, Kita Akita Municipal Hospital, Kita Akita; ${ }^{6}$ Orthopedic Surgery, Nakadori General Hospital; ${ }^{7}$ Orthopedic Surgery, Akita University Graduate School of Medicine, Akita, Japan

Background: The Akita Orthopedic Group on Rheumatoid Arthritis (AORA) encompasses 32 physicians and 28 clinics providing medical care to rheumatoid arthritis (RA) patients in Akita Prefecture, Japan. The patient registry for this group (the AORA registry) reflects actual clinical data on RA therapy in Akita, where the proportion of elderly (age $\geq 65$ years) residents in the population is the highest in Japan. Etanercept (ETN) is a tumor necrosis factor inhibitor reportedly associated with fewer adverse event-related treatment discontinuations than other drugs of this class. However, few evaluations of the efficacy and safety of ETN have been reported for elderly populations.

Objectives: Based on data from the AORA registry, we aimed to investigate the continuation rate for ETN therapy, reasons for discontinuation, and therapeutic effects among elderly RA patients living in Akita.

Methods: Among 204 AORA-registered patients starting ETN therapy between January 2009 and August 2014, data for the 73 patients (35.8\%) who were $\geq 65$ years old at the initiation of therapy were evaluated. Mean age was $72.4 \pm 4.7$ years (range $65-83$ years), and $79.5 \%$ were women. Mean disease duration was $15.1 \pm 12.6$ years (range 9 months- 55 years), $13.7 \%$ of patients were switching from another biologic agent, $49.3 \%$ could perform activities of daily living (ADL) independently, and $65.8 \%$ had at least one of the following comorbidities: hypertension, diabetes, respiratory disorder, cardiovascular disease, and cerebrovascular disease. We evaluated the 1-year cumulative continuation rate for ETN therapy using the Kaplan-Meier method, and investigated the characteristics of patients who discontinued treatment because of adverse events (AE cohort) or lack of efficacy (LOE cohort). We evaluated efficacy in 55 patients for whom Disease Activity Score - C-reactive protein assessments were possible, based on European League Against Rheumatism (EULAR) criteria.

Results: The 1-year cumulative continuation rate for ETN therapy was $87.2 \%$, and 24 patients discontinued treatment. The AE and LOE cohorts contained 12 and 7 patients, respectively. The AE cohort had a mean age of 75.5 years at the start of treatment, with a mean disease duration of 20.7 years, $8.3 \%$ of patients switching from another biologic agent, $16.7 \%$ performing ADL independently, and a comorbidity rate of $100 \%$. Corresponding values for the LOE cohort were: mean age, 71.4 years; disease duration, 12.7 years; switching from another biologic agent, 42.9\%; performing ADL independently, $57.1 \%$; and comorbidity rate, $28.6 \%$. Efficacy was noted for $81.8 \%$ of all patients with 52 weeks of ETN therapy, achieving good efficacy in 21 cases and moderate efficacy in 24 cases. Conclusions: Retention rate and efficacy were considered satisfactory in elderly RA patients receiving ETN therapy. The risk of adverse events was suggested to increase with increasing age, declining ADL, and presence of comorbidities. These factors require attention when prescribing ETN therapy.

\section{References:}

[1] Cho SK, Sung YK, Kim D, et al. Drug retention and safety of TNF inhibitors in elderly patients with rheumatoid arthritis. BMC Musculoskelet Disord. 2016; 17: 333 .

Disclosure of Interest: None declared

DOI: 10.1136/annrheumdis-2017-eular.6102

\section{AB0383 SUBCLINICAL BRAIN DAMAGE IN PATIENTS WITH RHEUMATOID ARTHRITIS AND ITS RELATIONSHIP TO TNF BLOCKER THERAPY}

S. Sağ, I. Tekeoğlu, M.S. Sağ, A. Kamanlı, K. Nas. Department of Physical Medicine and Rehabilitation, Rheumatology, Faculty of Medicine, Sakarya University, Sakarya, Turkey, sakarya, Turkey

Background: RA is a chronic disease with a yet unclarified etiology, which causes the activation of pro-inflammatory pathways that bring about joint and systemic inflammations (1).In recent years, the pathophysiology of brain damage that can occur in RA has drawn attention. Emphasis is being put on the possibility that brain damage occurs due to blood-brain barrier (BBB) damage that is linked to chronic inflammation

Objectives: In this study, we aimed to investigate the peripheral blood levels of brain-specific proteins such as $\mathbf{S} 100$ beta and GFAP (glial fibrillary acidic protein), the differences in these proteins in patients who did and did not undergo TNF blocker therapy and their relationship with cranial MR lesions, disease activity and cognitive functions with the purpose of determining CNS (central nervous system) damage in patients with rheumatoid arthritis (RA).

Methods: 58 RA patients $(47(81.0 \%)$ females, $11(19.0 \%)$ males) and 34 healthy controls $(24(70.6 \%)$ females, $10(29.4 \%)$ males) were included in the study. All RA patients were on synthetic DMARD therapy at the beginning. While 30 patients continued SDMARD therapy, 28 patients with high disease activity were started on TNF blocker therapy. All demographic characteristics of the patients were recorded. Disease activity was evaluated using DAS28. The Mini-Mental State Examination (MMSE) was used to evaluate cognitive functions, and the Fazekas Scale was used to assess the cranial MRI lesions. The peripheral blood S100 beta, GFAP, claudin, IL-17, IL-1 beta levels of the patients were measured at the beginning and on the 6th month.

Results: Demographic characteristics were similar between the two groups and no statistical difference was detected between the patient group and the control group in terms of sex, age, and BMI. ( $p>0.05)$ S100 beta and GFAP levels were higher to a significant degree compared to the control group. $(p<0.05)$ In the group that was started on TNF blocker therapy, S100 beta and GFAP levels were detected to have decreased significantly 6 months after treatment compared to the start of treatment. $(p<0.05)$ No difference was found between the RA and control groups in terms of hyperintense lesions seen in the cranial MRI. $(p>0.05)$ As the lesions in the deep white matter seen in the cranial MRI of RA patients increased, their $S 100$ beta levels were also seen to increase. $(p<0.05)$

Conclusions: In conclusion, next to decreasing disease activity and joint erosions by suppressing inflammation, anti-TNF therapy in RA can also suppress potential brain damage linked to subclinical BBB (blood-brain-barrier) dysfunction. Further studies with broader participation and longer patient follow-up are needed to reinforce this hypothesis.

References:

[1] McInnes, I.B. and Schett, G. (2011). Thepathogenesis of rheumatoidarthritis. N Engl J Med 365, 2205 2219.doi:10.1056/ NEJMra1004965.

Disclosure of Interest: None declared

DOI: 10.1136/annrheumdis-2017-eular.2315

\section{AB0384 CLINICAL AND RADIOLOGICAL EVOLUTION IN RHEUMATOID ARTHRITIS (RA) PATIENTS AFTER DEINTESIFICATE BIOLOGICS}

J. Uceda, R. Hernandez, J.L. Marenco. Rheumatology, Valme University Hospital, Seville, Spain

Background: RA is the most common chronic inflammatory arthritis. About $30 \%$ of patients are treated with biological therapy (BT). Deintensification of BT for patients in clinical remission, is a strategy used in clinical practice to reduce side effects and burden.

Objectives: The primary endpoint was evaluate clinical and radiological behavior of the RA in patients receiving BT at reduced doses. 
The secondary endpoint was to analyze the characteristics of patients who remain for a longer period of time in an optimized dose regimen.

Methods: In our Rheumatology Unit we are treating 271 RA patients with BT, the dose was deintensificated for $62(23 \%)$ patients in remission or low disease activity for at least 6 months. We have selected 32 patients with BT reduced for at least 6 years in an observational, descriptive, longitudinal and retrospective study. Disease activity was measured by the DAS 28 index. Structural damage was evaluated by SENS method.

Results: We analyze 32, 20 female, 12 male, mean age at diagnosis 42.6 years old; BT was started after RA evolution of 98.63 months. Drug reduction was performed after full BT for 62 months, mean DAS 28 was 2,47.

Patients were $75 \%$ FR positive and $56.7 \%$ ACPA positive. Etanercept was the BT more commonly reduced $59.4 \%$, followed by adalimumab $21.9 \%$, infliximab $12.5 \%$ and certolizumab $6.3 \%$.

BT dose returned to normal for 11 patients because of disease activity worsening after an average time of 15.90 months.

For 21 patients remaining on reduced doses, the mean DAS28 at time for analysis was 2.67.

BT reduction as different drugs: none infliximab reduced dose patients required return to normal dose. All certolizumab reduced (2 patients) patients needed to back to normal dose. Etanercept in 36,8\% and adalimumab 28,6\%.

The mean of SENS score before the optimization was 8.78 and at time for analysis 10.67 for both kind of patients, who continued reduced and those who needed to increase BT dose.

For the secondary endpoint 10 out of 12 male continue with deintensificated BT $(83 \%)$ in the other hand only 11 out of 20 female $(55 \%)$ maintained reduced dose.More negative for FR $(69,2 \%)$ and ACPA $(75 \%)$ patients keep on reduced dose regimen.

Conclusions: We have deintensificated 62 out of 271 RA patients on BT (23\%). All patients were in clinical remission at the beginning of BT dose reduction for more than 6 months.

Most patients $(65 \%)$ analyzed remain long time with reduced BT in clinical remission.

We have not observed significant X-ray progression for reduced patients, even if they increase disease activity and need to back to the original BT dose.

The increase in disease activity was the main reason to interrupt the optimization regime.

Infliximab was the drug that remained more time optimized.

According to the results of our study, male patients negative for ACPA and FR remain longer with reduced doses

Disclosure of Interest: None declared

DOI: 10.1136/annrheumdis-2017-eular.5273

\section{AB0385 STRATEGIES FOR THE OPTIMIZATION OF BIOLOGICAL THERAPY IN PATIENTS WITH JOINT INFLAMMATORY DISEASE: ANALYSIS OF CLINICAL RESULTS AT 4 YEARS}

M.J. Rodriguez Valls, M.D. Toledo Coello, Y. Cabello Fernandez, M.V. Perez Romera, J.J. Perez Venegas. Reumatologia, Hospital de Jerez, Jerez de la Frontera, Spain

Background: Biological therapies optimization in joint inflammatory diseases is indicated in patients who have more than six months in clinical remission. The main objective is to limit the occurrence of adverse effects, which are dosagedependent. In addition, the cost savings suppose a better access of new patients to these treatments. Given the increasing importance of this topic in recent years, we present the optimization experience in our center at the last 4 years

Objectives: 1.To analyze the clinical evolution evaluated by DAS28 in patients with rheumatoid arthritis (RA) and polyarticular psoriatic arthritis (PPSA) in biological therapy (BT) followed in a university hospital in the south of Spain which are performed in optimization of BT. 2. To analyze the optimization strategies used with the different $\mathrm{BT}$

Methods: Observational, longitudinal, retrospective and descriptive study by the review of clinical records, between January 2013 and January 2017, of patients with RA and PPSA who underwent BT optimization for more than 6 months at follow-up (DAS28 <2.6) or minimal activity (DAS28 2.6-3.2). We analyze demographic data, time of evolution of illness before the use of BT, time of TB until optimization, clinical evaluation by DAS28 and therapeutic strategies. Statistical analysis was performed with the IBM SPSS Statistics program

Results: From 294 patients in BT (174 RA and 120 PPsA), 95 (32.3\%) were submitted to optimization treatment: 58 in RA group and 37 in PPSA group. 57 were women and 38 men, with $56 \pm 12$ years mean age. The mean treatment time at optimized doses was $32 \pm 17$ months. At the optimization time, 85 (89.5\%) patients presented DAS28 remission and $10(10.5 \%)$ had low activity; at the study cut time, $67(70.5 \%)$ of them continue at clinical remission, $14(14.7 \%)$ low activity, $11(11.6 \%)$ moderate and $3(3.2 \%)$ high activity according to DAS28. A $69.5 \%$ (66) of the patients continued with optimized doses at the end of the study. From the 58 patients included in the RA group, 14 ( 5 with adalimumab $40 \mathrm{mg} / 21$ days, 8 with etanercept $25 \mathrm{mg} / 7-10$ days or $50 \mathrm{mg} / 10$ days and 1 with certolizumab $200 \mathrm{mg} / \mathrm{month}$ ) need the standard dose of the drug for disease control; a patient receiving etanercept discontinued the treatment after the diagnosis of breast cancer, as well as three other patients treated with adalimumab who were diagnosed of pancreatic cancer, septic arthritis and cognitive impairment respectively. On the other hand, 9 patients from of the PPSA group needed to restart the treatment at standard doses, either for articular (4) or cutaneous (5) worsening, 3 of them being treated with adalimumab and 6 with etanercept. In addition, a patient had to be discontinued due to the diagnosis of decompensated liver cirrhosis; another patient suspended it voluntarily

Conclusions: After 4 years, we can conclude that in our cohort of patients with inflammatory joint disease in BT that have been submitted to dose optimization because they were in remission or low disease activity, a high percentage $(69.5 \%)$ remain in the same clinical situation for an average of 32 months. The response rate obtained for optimization in the RA group and in the PPSA group are comparable. The most frequent optimization strategies employed were adalimumab $40 \mathrm{mg} / 21$ days, etanercept $25 \mathrm{mg} / 7$ days and etanercept $50 \mathrm{mg} / 10$ days.

Disclosure of Interest: None declared DOI: 10.1136/annrheumdis-2017-eular.4326

\section{AB0386 A STUDY OF RETENTION AND SWITCHING RATES OF 1ST LINE BIOLOGICS FOR RHEUMATOID ARTHRITIS}

M. Sato, T. Tani, T. Ohashi. Rheumatology, Ohashi and Tani Orthopedics, Gifu, Japan

Background: Since the introduction of biologics for rheumatoid arthritis (RA) treatment, significant improvements in joint inflammation control, prevention of bone/joint destruction, activities of daily living ( $A D L)$, and quality of life (QOL) have been observed. Tumor necrosis factor (TNF) inhibitors, such as anti-TNF drugs and TNF receptor drugs, accounted for the majority of biologics. However, the use of non-TNF inhibitors, including interleukin (IL)-6 receptor antibodies and selective modulators of T-cell co-stimulation, has been approved, and their efficacy has been demonstrated.

Objectives: This study aimed to analyze the effective usage retention rate of TNF inhibitors and non-TNF inhibitors in RA patients.

Methods: Among 475 RA patients who were administered biologics up to the end of 2015, 329 patients who had been treated for more than 5 years (treatment initiated before the end of December 2010) since the first introduction of biologics (1st line) were included in this study. These patients were divided into TNF inhibitor (infliximab: IFX, etanercept: ETN, and adalimumab: ADA) and non-TNF inhibitor groups (tocilizumab: TCZ, and abatacept: ABT) to investigate the number of patients who progressed to 2 nd or 3 rd line therapy by 5 years after introduction, and which biologic was administered as the 2nd or 3rd line therapy in each group. Results: Of 329 patients, 67 were men and 262 were women. Patient age ranged between 22 and 83 years with a mean of $58.7 \pm 13.5$ years. RA disease duration ranged between 1 and 50 years with a mean of $9.6 \pm 8.1$ years. TNF inhibitors and non-TNF inhibitors were used as 1st line therapy in 278 and 51 patients respectively. In the TNF inhibitor group, IFX, ETN, and ADA were administered to 145,87 , and 46 patients respectively. In the non-TNF inhibitor group, TCZ and ABT were administered to 48 and 3 patients respectively. In the TNF inhibitor group, 94 of 278 patients (33.8\%) progressed to 2 nd line therapy owing to efficacy attenuation and adverse events. Thirty-four of these patients were switched to TNF inhibitors and 60 to non-TNF inhibitors. Conversely, 6 of 51 patients in the non-TNF inhibitor group (11.8\%) advanced to 2nd line therapy, with 2 switching to TNF inhibitors and 4 to non-TNF inhibitors. Additionally, 25 and 3 patients advanced to 3rd line therapy in the TNF inhibitor and non-TNF inhibitor groups respectively. Throughout the 5 years, 154 (55.4\%) patients in the TNF inhibitor group did not change their treatment agent while $69(24.8 \%), 24(8.6 \%)$, and $1(0.4 \%)$ switched once, twice, or 3 times or more, respectively. Thirty $(10.8 \%)$ patients discontinued biologic usage. In the non-TNF inhibitor group, 3 (5.9\%), $2(3.9 \%)$, and $1(2.0 \%)$ patients switched once, twice, or 3 times, respectively. Six $(11.8 \%)$ patients discontinued biologic usage, and no change in treatment occurred in $39(76.5 \%)$ patients. The mean number of biologic agent switches per patient in each group over 5 years was 0.43 times in the TNF inhibitor group and 0.20 times in the non-TNF inhibitor group, indicating that the number was significantly lower in the non-TNF inhibitor group $(p=0.0032)$.

Conclusions: While the sample size was small and patient characteristics varied, it appears that non-TNF inhibitors are not inferior to TNF inhibitors as 1st line therapy biologics in terms of retention rate and number of switches to TNF inhibitors. Disclosure of Interest: None declared

DOI: 10.1136/annrheumdis-2017-eular.3356

\section{AB0387 COMPARISON OF ETANERCEPT IN MONOTHERAPY AND COMBINATION WITH SYNTHETIC DMARDS: DATA FROM ATTRA REGISTRY}

P. Horák ${ }^{1}$, M. Skácelová ${ }^{1}$, L. Szczuková ${ }^{2}$, A. Smržová ${ }^{1}$, J. Vencovský $^{3}$ J. Závada ${ }^{3}$, K. Pavelka ${ }^{3}$ on behalf of ATTRA registry Czech Republic. ${ }^{1}$ III Department of internal Medicine, Faculty of Medicine and Dentistry, University Olomouc, Olomouc; ${ }^{2}$ Institute of Biostatistics And analysis, Masaryk University Brno, Brno; ${ }^{3}$ Institute of Rheumatolgy, Charles University of Prague, Prague, Czech Republic

Background: Etanercept (ETN) is an established bDMARDs for therapy of rheumatoid arthritis and some other inflammatory diseases. In rheumatoid arthritis patients ETN could be given in combination therapy with sDMARDs as well as in monotherapy. 